\title{
Biomechanical evaluation of wrist-driven flexor hinge orthosis in persons with spinal cord injury
}

\author{
Yeoun-Seung Kang, MD, PhD, CPO; ${ }^{1}$ Yoon-Ghil Park, MD, PhD $;^{2}$ Bum-Suk Lee, MD ${ }^{3}$ Hyung-Soon Park, \\ $\mathbf{P h D}^{1,4^{*}}$ \\ ${ }^{1}$ Department of Rehabilitation Medicine, Clinical Center, National Institutes of Health, Bethesda, MD; ${ }^{2}$ Gangnam \\ Severance Hospital, Yonsei University College of Medicine, Seoul, Korea; ${ }^{3}$ National Rehabilitation Hospital, Seoul, \\ Korea; ${ }^{4}$ Department of Mechanical Engineering, Korea Advanced Institute of Science and Technology, Daejeon, Korea
}

\begin{abstract}
The wrist-driven flexor hinge orthosis (WDFHO) is a device used to restore hand function in persons with tetraplegic spinal cord injury by furnishing three-point prehension. We assessed the effectiveness and biomechanical properties of the WDFHO in 24 persons with cervical 6 or 7 tetraplegia who have severely impaired hand function. This study introduces a mechanical operating model to assess the efficiency of the WDFHO. Experimental results showed that pinch force increased significantly $(p<0.001)$ after using the WDFHO and was found to positively correlate with the strength of wrist extensor muscles $(r=0.41, p<0.001)$. However, when the strength of the wrist extensors acting on the WDFHO was greater, the reciprocal wrist and finger motion that generates three-point prehension was less effective $(r=0.79, p<0.001)$. Reliable and valid biomechanical evaluation of the WDFHO could improve our understanding of its biomechanics.
\end{abstract}

Key words: biomechanics, efficiency, finger motion, hand function, mechanical operating model, pinch force, spinal cord injury, three-point prehension, wrist-driven flexor hinge orthosis, wrist extensor muscles.

\section{INTRODUCTION}

Promoting functional hand activities is a crucial rehabilitation goal for persons with tetraplegic spinal cord injury (SCI) [1-4]. Some people with mid- to low-level cervical (C) SCI achieve useful tenodesis grasp, which is opposition of the thumb and the index and middle fingers through reciprocal wrist extension and finger flexion, with the aid of a wrist-driven flexor hinge orthosis (WDFHO) [5-6]. Generally, persons with C6 and C7 tetraplegia can use a WDFHO for a variety of daily activities, such as eating, dressing, using the toilet, grooming, and writing $[4-5,7]$. The WDFHO enables persons with tetraplegic SCI to attain a functional tenodesis grasp that creates enough passive tension in the paralyzed thumb and finger flexor muscles (such as flexor pollicis longus, flexor digitorum superficialis, and flexor digitorum

\footnotetext{
Abbreviations: AIS $=$ American Spinal Injury Association Impairment Scale, $\mathrm{C}=$ cervical, $\mathrm{CO}_{2}=$ carbon dioxide, $\mathrm{ECRB}=$ extensor carpi radialis brevis, ECRL = extensor carpi radialis longus, ECU = extensor carpi ulnaris, EMG = electromyography, $\mathrm{FES}=$ functional electrical stimulation, $\mathrm{FHO}=$ flexor hinge orthosis, IP = interphalangeal, $\mathrm{MCP}=$ metacarpophalangeal, $\mathrm{MMT}=$ manual muscle test, $\mathrm{MVC}=$ maximum voluntary contraction, $\mathrm{ROM}$ = range of motion, $\mathrm{SCI}=$ spinal cord injury, $\mathrm{WDFHO}=$ wrist-driven flexor hinge orthosis.

*Address all correspondence to Hyung-Soon Park, PhD; Department of Mechanical Engineering, Korea Advanced Institute of Science and Technology, 291 Daehak-ro, Yuseong-gu, Daejeon, 305-701, Republic of Korea; +82-42350-3038; fax: +82-42-350-3210.

Email: hvungspark@kaist.ac.kr http://dx.doi.org/10.1682/JRRD.2012.10.0189
} 
profundus) with active wrist extension by contraction of the innervated extensor carpi radialis brevis (ECRB) and extensor carpi radialis longus (ECRL). Therefore, the WDFHO is an ideal device for persons with $\mathrm{C} 6$ or $\mathrm{C} 7$ tetraplegia who have wrist extensors with muscle strength of grade 3 or above on the manual muscle test (MMT) and who have either flickers or no finger movement to furnish their prehension effectively [8].

The WDFHO is made up of three main components: finger, palmar, and forearm. These three components are hinged at the anatomical axes of the wrist and metacarpophalangeal (MCP) joints. The WDFHO operates on a two-hinge parallelogram system that converts actively controlled wrist extension to passive flexion of the fingers at the MCP joint, resulting in a three-point prehension or three-jaw chuck grasp in which the index and middle fingers move together toward the immobilized thumb. Conversely, when the wrist flexes actively or passively due to gravity, the MCP joint extends and the fingers open. An adjustable actuating lever at the wrist joint determines the level of wrist extension, allowing the hand to open and close at varying degrees. The level of wrist extension needed is based on the size of the object to be grasped.

The flexor hinge orthosis (FHO) was originally designed to restore upper-limb function of persons with poliomyelitis [9]. As the incidence of poliomyelitis decreased, researchers began to investigate the application of FHO to other populations with upper-limb paralysis, such as SCI, hemiplegia, and brachial plexus injury [9-10].

Variations of FHOs exist for persons with SCI who are unable to use the wrist extension functionally [11-16]. Persons with high-level SCI who lack voluntary wrist extension and hand motion are suitable candidates for the ratchet FHO, the Mckibben FHO, the electric motordriven FHO, and the shoulder harness-driven FHO.

The ratchet FHO is designed to push a ratchet lever until the fingers reach the desired position [11]. To form a three-point prehension, the user must exert force on the lever to passively close the fingers by gross motion of the other hand or by pushing against any stationary object. When the ratchet button is tapped, the ratchet lock is opened and the grasp is released.

Two external power sources, compressed carbon dioxide $\left(\mathrm{CO}_{2}\right)$ gas or an electric motor, can also substitute for paralyzed wrist extensors in a second variation of the FHO. The $\mathrm{CO}_{2}$ gas is used to inflate an artificial mus- cle, also referred to as the Mckibben muscle, which consists of an inner rubber tube and an outer helically woven fabric [13]. When pressurized with $\mathrm{CO}_{2}$, this flexible rubber "bladder" expands against the woven fabric and shortens in length like a real muscle. Inflation of the artificial muscle propels the fingers into flexion against the stable thumb, and grasp release after deflating the Mckibben muscle can be achieved by gravity, spring, or the pull of a rubber band. The electrical motor-driven FHO is powered by a rechargeable battery pack [14-16]. It can be controlled by a switch, which the patient may activate using any available muscle. The electromyography (EMG) signal can proportionally control the electric motor using a microprocessor.

Finally, the Bowden cable system can be incorporated to activate three-point prehension in the shoulder harness-driven FHO [12]. In this orthosis, the shoulder motion pulls the cable out of its housing and transfers power from the shoulder musculature to the FHO to release the grasp. Grasp closure is produced by a rubber band or flexor spring pull.

This study excludes these four FHO variations because of their complexity [17]. These orthoses are subject to actuators' bulkiness, poor cosmesis, and donning issues and require more technical support and more sophisticated training programs than the WDFHO prototype operated by voluntary wrist extension.

Until now, other rehabilitative approaches also have been attempted to improve hand function in persons with tetraplegic SCI [18-25]. Functional electrical stimulation (FES) enables persons with tetraplegia to restore grasp function [18-22]. This system uses electrical current pulses to excite nerves going to paralyzed muscles and provokes contraction of the muscles required for grasp and release. In addition, reconstructive surgery can be performed to transfer active muscles or shorten tendons to enhance grasp force [23-25].

Despite the emergence of new technologies and evolution of upper-limb rehabilitation for persons with tetraplegia, the WDFHO still continues to be used to improve hand function because of its noninvasiveness, simplicity of design, relatively low cost, and easy availability in the market [17-18,20]. However, little is known about the biomechanical properties of this orthosis. Most of the available studies for the WDFHO date from the 1960s to the mid-1980s and only discuss basic construction and structural modifications [9,26-27]. Furthermore, the majority of information on using the WDFHO in clinical 
practice comes from protocols that various departments had written for their own use [28-31], and clinical reports in the literature rely heavily on questionnaires to evaluate the efficacy of the WDFHO [5-6]. Thus, it is difficult to accurately assess the usefulness of the WDFHO and to clearly understand its function without a careful biomechanical analysis involving mechanical modeling of the operating principle. Given the limited information on biomechanical characteristics in the literature, additional biomechanical assessment for the WDFHO is imperative. The overall goal of this study is to evaluate the effectiveness of the WDFHO by providing quantitative biomechanical analysis of the orthosis.

\section{METHODS}

\section{Subjects}

We recruited 24 persons with complete SCI ( 22 male and 2 female, $37.1 \pm 12.8$ yr old [mean \pm standard deviation]) who have C6 or C7 American Spinal Injury Association Impairment Scale (AIS) grade A [32] and paralysis or severe weakness of the hands. AIS classifica- tion was determined by medical chart review. Each patient had been injured for $5.6 \pm 7.3 \mathrm{yr}$ at the time of the study. To be eligible for participation, patients must have scored at least grade $3(\geq 3 / 5)$ on an MMT for wrist extensor muscles. Patients with spasticity or contracture that interferes with hand grasp function were excluded.

\section{Wrist-Driven Flexor Hinge Orthosis}

Study subjects were fitted with an adjustable WDFHO (Talon ${ }^{\mathrm{TM}}$, North Coast Medical Inc; Gilroy, California) consisting of a polyethylene forearm and a palmar cuff to grasp objects. This orthosis is a prefabricated device (Figure 1). The lengths of links $L_{1}$ and $L_{3}$ (Figure 1(b)) are adjustable to fit each subject from the radial side of the second MCP joint to the distal tip of the radial styloid and to match the subject's available range of motion (ROM) at the MCP joint. The interphalangeal (IP) joints of the index and middle fingers are stabilized along with the IP and MCP joints of the thumb. When a subject extends his or her wrist, the posted thumb and index and middle fingers are pushed together to attain a grasp motion. Conversely, wrist flexion causes the hand to open. There are five levels in the gear slot selector that (a)

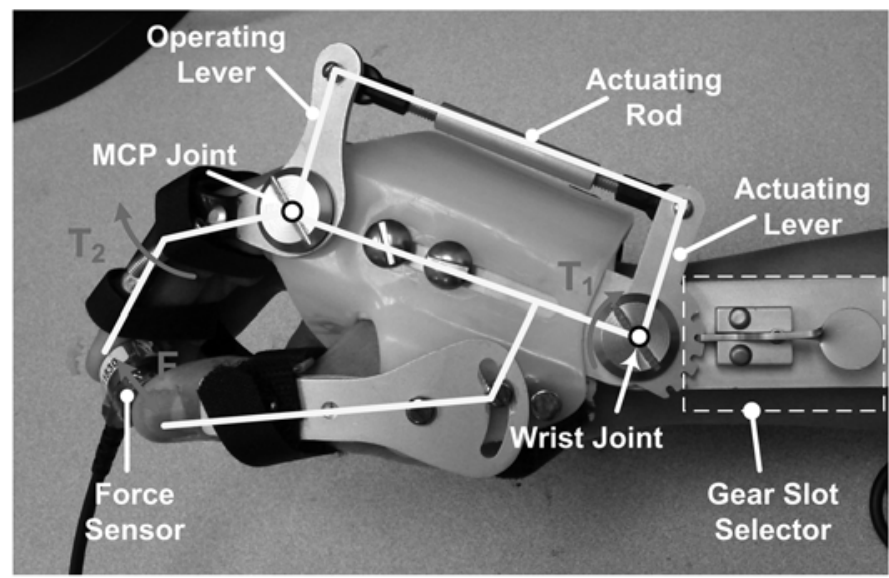

(b)

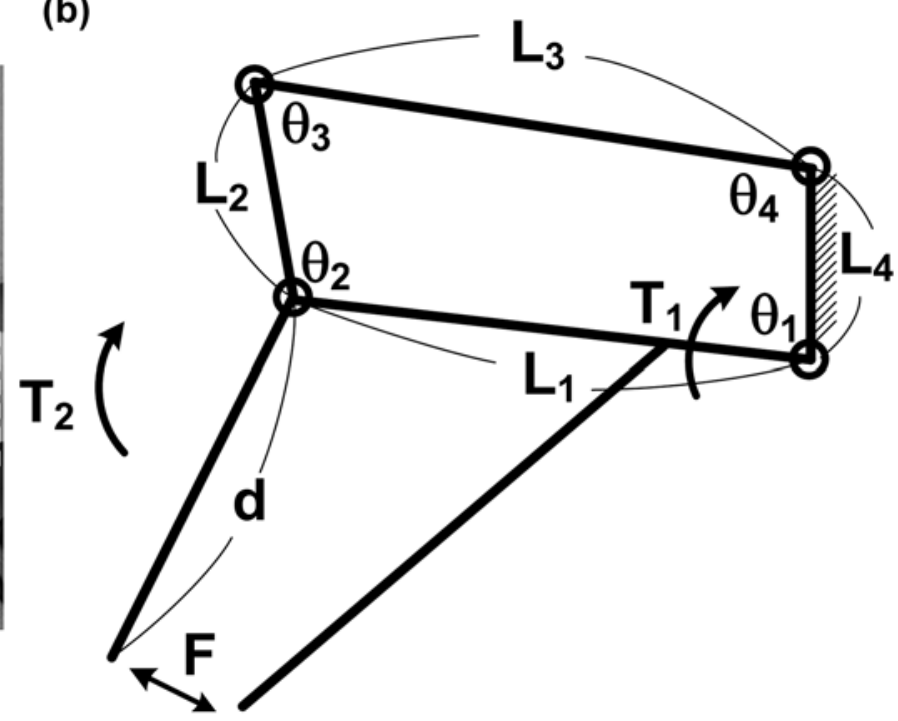

Figure 1.

(a) Wrist-driven flexor hinge orthosis (WDFHO) structure and three-point prehension in example of right-hand side. (b) Schematic diagram of grasp motion with WDFHO. $d=$ length from radial side of second MCP joint to fingertip, $F=$ three-point pinch force, $L_{1}=$ length from radial side of second MCP joint to distal tip of radial styloid, $L_{2}=$ length of operating lever, $L_{3}=$ length of actuating rod, $L_{4}=$ length of actuating lever, MCP $=$ metacarpophalangeal, $T_{1}=$ wrist extension torque, $T_{2}=$ MCP joint torque, $\theta_{1}-\theta_{4}=$ angles between four linkages. 
regulate the angle of wrist extension (Figure 1(a)). Pressing the spring-loaded button of the gear slot selector locks the notched actuating lever into the desired position. A certified orthotist and occupational therapist set up the WDFHO for each subject throughout the experiment.

Figure 1 shows the WDFHO structure and corresponding schematic diagram for deriving static governing equations. In a typical WDFHO structure, the wrist extension torque $\left(T_{1}\right)$ is transferred to the MCP joint through the four bar linkages system. The wrist extension torque $\left(T_{1}\right)$ rotates link $1\left(L_{1}\right)$ clockwise, which results in a counter-clockwise rotation of the operating lever $\left(L_{2}\right)$. The resultant torque at the MCP joint $\left(T_{2}\right)$ is balanced with the three-point pinch force $(F)$ at the static pinch. The torque at the MCP joint $\left(T_{2}\right)$ is determined from the geometry of the four bar linkages as the following (Equation (1)):

$$
T_{2}=\frac{L_{2} \cos \left(90^{\circ}-\theta_{3}\right)}{L_{2} \cos \left(90^{\circ}-\theta_{3}\right)+L_{1} \sin \left(\theta_{1}+\theta_{4}\right)} T_{1}
$$

See Appendix (available online only) for detailed derivation.

Since $T_{2}$ is equivalent to the pinch force $(F)$ multiplied by the moment arm (d), the resultant pinch force is calculated as the following (Equation (2)):

$$
F=\frac{L_{2} \cos \left(90^{\circ}-\theta_{3}\right)}{L_{2} \cos \left(90^{\circ}-\theta_{3}\right)+L_{1} \sin \left(\theta_{1}+\theta_{4}\right)} \times \frac{T_{1}}{d} \text {. }
$$

\section{Evaluation Procedures}

Subject hand sizes (lengths of thumb and fingers and lengths between wrist to MCP joints of thumb and fingers) were measured prior to the test in order to fit the WDFHO. A physician measured passive and active ROM of the wrist and hand joints [33] and assessed the strength of wrist and hand muscles by using a MMT. Subjects were seated in their own wheelchairs and positioned into the most upright posture possible (hip, knee, and ankle joints angles at $90^{\circ}$ ). The subjects' dominant upper limbs were supported on the table with their shoulders adducted and neutrally rotated, elbow flexed at $90^{\circ}$, forearm in

\footnotetext{
*The direction of rotations refers to the example of right-hand side. The motions are opposite in the left-hand side.
}

fully pronated position, wrist at $0^{\circ}$ extension, and no radial or ulnar deviation [34-35]. A cushion (2 in. thick) was placed under the wrist to provide enough room to flex the wrist joint. Two surface EMG electrodes (Shimmer; Dublin, Ireland) were attached two fingerbreadths distal to the lateral epicondyle [36] to monitor ECRB and ECRL muscle activity during wrist extension. The threepoint pinch force of each subject's dominant hand without the WDFHO was measured using a six degrees of freedom force transducer (Nano17 force/torque sensor, ATI Industrial Automation; Apex, North Carolina) (Figure 2(a)). The WDFHO was fitted to the subject's dominant hand, and the wrist was extended to a selected level of the gear slot so that the subject could apply a maximum three-point pinch force while the force transducer measured the force (Figure 2(b)). The maximum voluntary contraction (MVC) of the subject's wrist extensors was measured using a custom-made dynamometer, which consists of forearm and hand cuffs and a torque transducer (TRT 100, Transducer Techniques Inc; Temecula, California) (Figure 2(c)). Each measurement was repeated three times, and the mean values were selected as the pinch force and MVC of the wrist extensors.

\section{RESULTS}

The mean pinch force of all 24 subjects was $0.64 \pm$ $0.42 \mathrm{~N}$ without using the WDFHO. The MMT for all hand muscles were grossly scored from grade 0 to 1 . However, the MMT for wrist extensors were scored as at least grade 3 for all subjects. Of the 24 subjects, 14 were scored as grade 4 (wrist extension MVC: $1.92 \pm 0.82 \mathrm{Nm}$ occurred at $29.4^{\circ} \pm 11.5^{\circ}$ wrist extension), 5 subjects had grade $\geq 3$ (wrist extension MVC: $0.71 \pm 0.03 \mathrm{Nm}$ occurred at $9.8^{\circ} \pm 16.7^{\circ}$ wrist extension), and 5 subjects had grade 3 (wrist extension MVC: $0.46 \pm 0.06 \mathrm{Nm}$ occurred at $26.4^{\circ} \pm 15.0^{\circ}$ wrist extension).

The pinch force while using the WDFHO was calculated to be $7.26 \pm 3.48 \mathrm{~N}$, which is $14.3 \pm 8.6$ times greater $(p<0.001)$ than the pinch force without the orthosis. The pinch force was significantly increased with the WDFHO, and greater MVC resulted in a greater pinch force increment $(r=0.41$, Figure 3(a)).

Ideally, the wrist extension MVC $\left(T_{1}\right)$ should be transferred to the pinch force $(F)$ as defined by Equation (2). However, due to the friction and misalignment, $T_{1}$ was not fully transferred to the pinch force. Torque transfer 


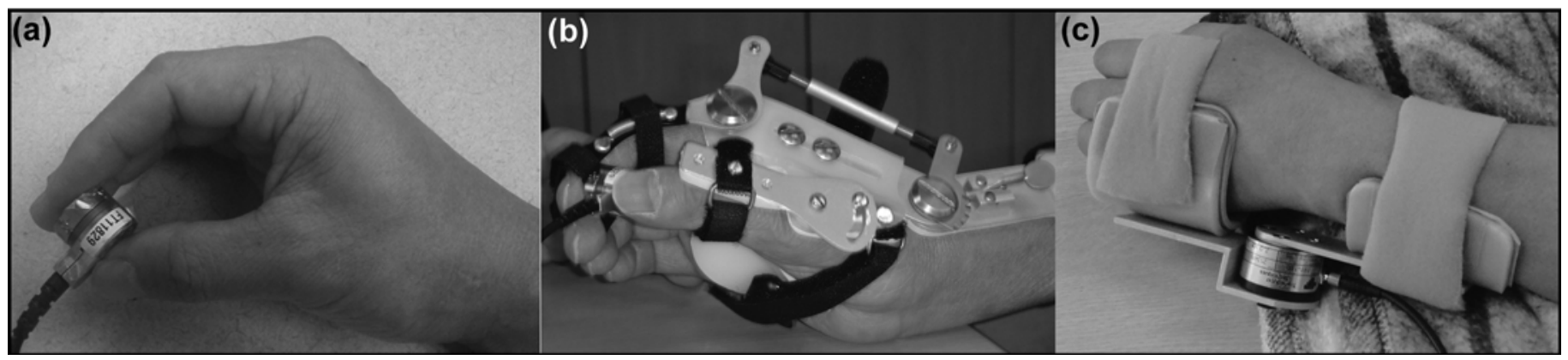

Figure 2.

Experimental procedures: (a) subject performing three-point pinch without using wrist-driven flexor hinge orthosis (WDFHO), (b) subject performing three-point pinch using WDFHO, and (c) subject performing wrist extension maximum voluntary contraction measurement task.

efficiency was calculated by dividing the actual pinch force measured with the WDFHO by the ideal pinch force calculated from the wrist MVC measurement. The ideal pinch force was calculated from Equation (2). The average efficiency was 37.6 percent, and the efficiency was greater for those subjects who had weaker wrist extensor strength $(r=0.79$, Figure 3(b)). However, the pinch efficiency was not significantly correlated with the wrist extension angle at MVC $(r=0.07)$.

\section{DISCUSSION}

The goal of this study was to perform an in-depth biomechanical evaluation of the WDFHO in persons with tetraplegic SCI. The results of this study differ distinctly from those of a similar study by Stenehjem et al. [37]. In the prior study, the investigators provided a hypothetical description of the torque ratio $\left(T_{2} / T_{1}\right)$ to evaluate the theoretical pinch force. However, a percent efficiency of the WDFHO in our study, calculated using their equation, was unrealistically high (nearly 364\%). In addition, the small subject sample size found in Stenehjem et al. was not statistically significant enough to support the use of the WDFHO. These study features led us to revisit the mechanical analysis of the WDFHO mechanism to determine new mathematical equations to describe its operating principle.

As expected, the WDFHO increased the pinch force markedly in all subjects. Moreover, greater MVC in wrist extensors resulted in greater pinch force. However, the efficiency of the WDFHO decreased as the MVC of wrist extensors increased. This finding suggests that stronger wrist extensor musculature results in lower efficiency of the reciprocal wrist and finger motion by using a WDFHO. To elucidate the negative correlation between the MVC and the efficiency of the WDFHO, we consider the influence of wrist musculature on the mechanical joint axes alignment of the WDFHO.

As the radial wrist extensors (ECRB and ECRL) receive the innervation from a higher level of the spinal cord, they often remain stronger than the ulnar wrist extensor, or extensor carpi ulnaris (ECU), in persons with C6 and C7 tetraplegia [38]. Thus, a strong tendency exists to drive the wrist into radial deviation as it is extended [6]. Conventionally, it is acknowledged that the mechanical joints of wrist and index finger in the WDFHO should be located at the distal tip of the radial styloid and the radial side of the second MCP joint, respectively [37]. If the WDFHO is fitted on a hand controlled by a weak wrist extensor musculature, the mechanical joints of the WDFHO are prone to coincide with the anatomical joints during the wrist ROM because the ECRB and ECRL muscles are not strong enough to concur with radial deviation. Thus, wrist movement from flexion to extension in weaker wrist extensors does not create too much deviation between anatomical and mechanical joints axes. In contrast, if the wrist extensors are stronger, the extension and radial deviation wrist movements cause the WDFHO to twist forcefully. Therefore, even when the WDFHO is accurately placed on the anatomical joint axes, the initial alignment could not be maintained. Consequently, the incongruency between anatomical and mechanical joint axes due to the combined wrist motions of stronger wrist extensor musculature in both sagittal and frontal planes 


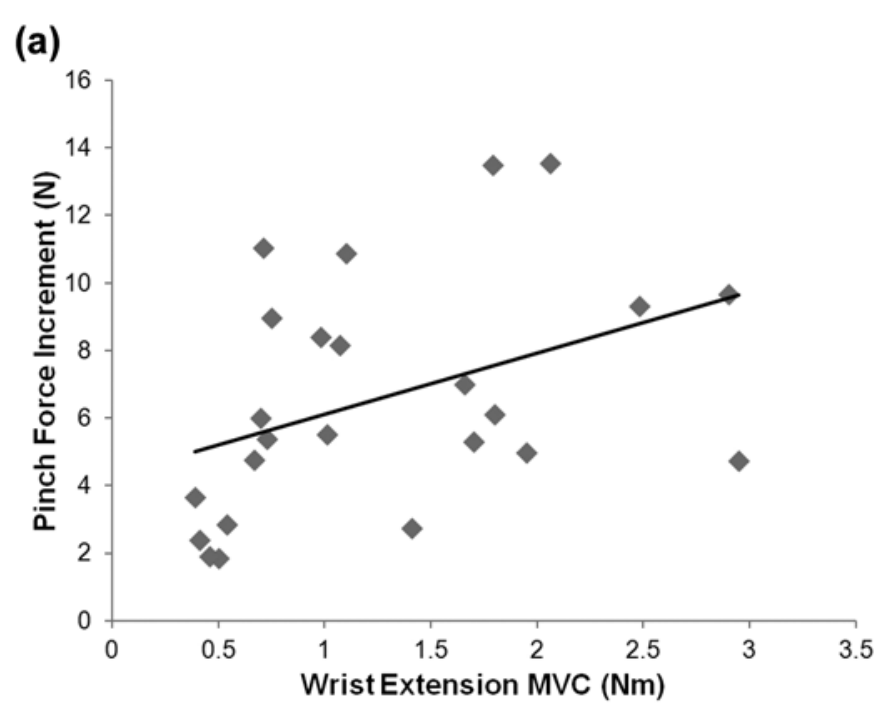

(b)

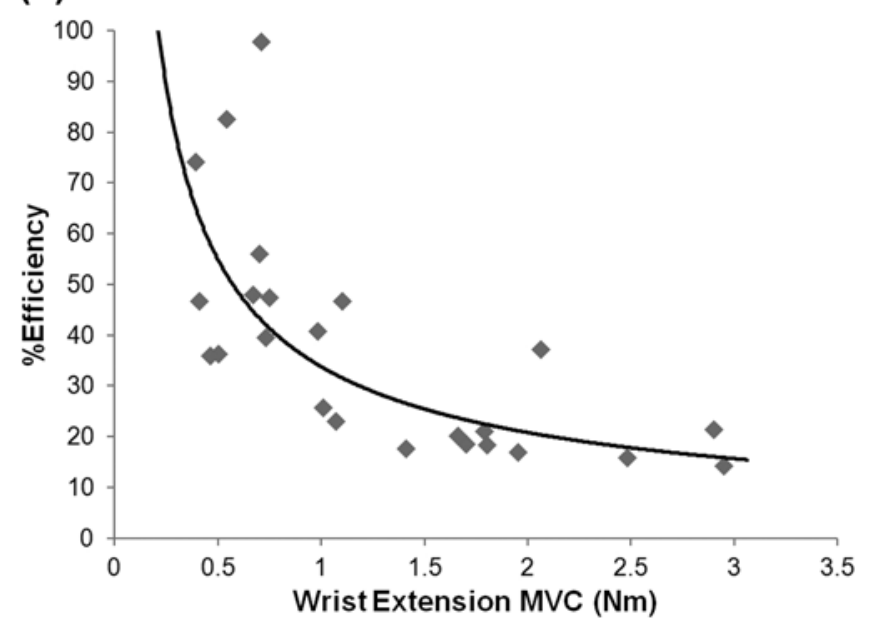

Figure 3.

(a) Relationship between wrist extension maximum voluntary contraction (MVC) and pinch force increment using wrist-driven flexor hinge orthosis (WDFHO) $(r=0.41, p<0.001)$, and (b) relationship between wrist extension MVC and percent efficiency of WDFHO $(r=0.79, p<0.001)$.

results in a reduced ability to transmit the wrist torque to pinch force during the tenodesis grasp.

Consequences of orthotic misalignment in the lower limbs have been revealed by previous studies [39-40]. These studies noted that a distorted orthotic alignment results in undesirable shear and/or compressive forces and moments that may affect the operating mechanism of the articulated lower-limb orthoses. Regarding the orthotic management of the nonfunctional hand, Perry stated that power loss results from the wrist traveling an oblique path while the WDFHO must be aligned longitudinally to match the anatomical axes of the wrist and MCP joints [41]. Furthermore, Johnson observed radial deviation with use of the WDFHO and proposed a specifically designed radial deviation WDFHO [38]. This conceptual design modification allows the WDFHO to track with the hand as it enters radial deviation without compromising pinch force. However, clinical application of this modified WDFHO is still at the experimental stage.

In addition to the previous findings pertaining to orthotic misalignment [38-41], the current study acknowledges that the reciprocal wrist extension and finger flexion of the stronger wrist extensor musculature is less effective than that of the weaker wrist extensor musculature when using a WDFHO. This finding may provide insights into designing a more effective WDFHO. For example, maintaining correct alignment of the WDFHO in persons with tetraplegia who have stronger wrist extensor musculature may require the development of a new total contact polymer WDFHO that ensures an intimate fit.

The unbalanced radial deviation of the wrist that restricts the efficient application of WDFHO might be solved by surgical procedures. Recently, a reconstructive hand surgery for rebalancing the radial deviation deformity of the wrist was introduced to help optimize grip strength in persons with tetraplegia [25]. Our findings agree with the need for the recently proposed surgical intervention, which can be a solution for achieving the balanced wrist extension, obtaining more efficient tenodesis grasp with WDFHO.

The FES system can also be a feasible solution for resolving the radial wrist extensors bias [18,21-22]. Thorsen et al. devised a myoelectrically controlled single-channel FES system to reinforce the tenodesis grasp in persons with C6 and C7 tetraplegia and found an imbalance of hand grasping muscles that resulted in reducing the grasp capability [18]. The limitation reported in the single-channel FES system gives us insight that more efficient tenodesis grasp can be achieved by combining the WDFHO with an FES system that stimulates ECU to counteract the undesirable radial deviation caused by stronger ECRB and ECRL in persons with $\mathrm{C} 6$ and $\mathrm{C} 7$ tetraplegia.

The wrist extension angle at MVC was not strongly correlated with the pinch efficiency in this study. Unlike the normal wrist extension angle range $\left(20^{\circ}-30^{\circ}\right)$ 
required for optimal grasp activities in nondisabled people [42-43], individual subject's wrist angle for optimal grasp varied largely $\left(23.6^{\circ} \pm 15.9^{\circ}\right)$, and this might be the reason for the weak correlation between the wrist extension angle and the efficiency.

Only the three-point prehension was assessed in this study because the thumb component of the WDFHO was positioned palmar to the index and middle fingers. However, it would be beneficial to evaluate the lateral pinch force in future studies. To perform a lateral pinch with the WDFHO, lateral rotation of the thumb component may be needed to allow the thumb to adduct to the radial side of the index finger. When the tenodesis grasp is achieved, persons with tetraplegia can grasp objects using a threepoint prehension or lateral pinch. Three-point prehension occurs when the pulp of the thumb is opposed to the pulps of the index and middle fingers. However, most persons with tetraplegic SCI who are able to operate a WDFHO can perform the lateral pinch, which occurs between the volar surface of the thumb and the side of index finger [44]. In addition, the pressure of thumb pulp against the lateral aspect of the index finger can be an effective means of obtaining tenodesis grasp for thin or flat objects and may be a more realistic rehabilitation goal for persons with severely impaired hand function.

In this study, we adjusted the actuating lever to a level where the subjects could obtain a maximal pinch force. The adjustable linkage between the wrist and fingers permits prehension to occur with the wrist in five positions. This allows the WDFHO users to select the most comfortable and functional wrist angle for specific activities. When the actuating lever is in the lowest slot, the hand opening is smaller and high-force pinching is accomplished with the wrist slightly flexed. This position is useful for fine activities such as picking up small objects. With the lever in the topmost slot, the hand opening is wider and firm prehension is attained with the wrist in extended position. This position is adequate for grasping large objects such as a cup. Our study did not investigate the relationship between wrist excursion and types of prehension. Further information on determining the optimal actuating lever settings for specific tasks will be beneficial for clinical application of the WDFHO.

\section{CONCLUSIONS}

This study evaluated the effectiveness of the WDFHO in persons with tetraplegic SCI. Our results showed that the pinch force was significantly higher with the application of the WDFHO, suggesting that the WDFHO is an effective device for improving tenodesis grasp. Moreover, the mechanical operating model of the WDFHO allowed us to quantitatively evaluate the efficiency of the orthosis, indicating that the pinch efficiency of the WDFHO decreased in the stronger wrist extensors. This finding highlighted the clinical importance of orthotic alignment. Overall, data from this study may potentially be used to better understand the biomechanics of the WDFHO with regard to design, fabrication, prescription, and fitting and may also enhance prehension capabilities in the rehabilitation of persons with severely impaired hand function.

\section{ACKNOWLEDGMENTS}

\section{Author Contributions:}

Study concept and design: Y.-S. Kang, H.-S. Park. Acquisition of data: Y.-S. Kang, H.-S. Park.

Analysis and interpretation of data: Y.-S. Kang, H.-S. Park.

Drafting of manuscript: Y.-S. Kang, H.-S. Park.

Critical revision of manuscript for intellectual content: Y.-S. Kang, H. -S. Park.

Statistical analysis: H.-S. Park.

Administrative, technical, or material support: Y.-G. Park, B.-S. Lee.

Financial Disclosures: The authors have declared that no competing interests exist.

Funding/Support: This material was based on work supported by the Intramural Research Program of the National Institutes of Health Clinical Center.

Institutional Review: This project was approved by the Institutional Review Board at the Gangnam Severance Hospital of Yonsei University College of Medicine, and all subjects signed an informed consent before participating in the study.

Participant Follow-Up: The authors do not plan to inform participants of the publication of this study because of a lack of contact information.

\section{REFERENCES}

1. Johanson ME, Murray WM. The unoperated hand: The role of passive forces in hand function after tetraplegia. Hand Clin. 2002;18(3):391-98. [PMID:12474591] http://dx.doi.org/10.1016/S0749-0712(02)00035-5 
2. van Tuijl JH, Janssen-Potten YJ, Seelen HA. Evaluation of upper extremity motor function tests in tetraplegics. Spinal Cord. 2002;40(2):51-64. [PMID:11930877]

http://dx.doi.org/10.1038/sj.sc.3101261

3. Rudhe C, van Hedel HJ. Upper extremity function in persons with tetraplegia: Relationships between strength, capacity, and the spinal cord independence measure. Neurorehabil Neural Repair. 2009;23(5):413-21.

[PMID:19261766] http://dx.doi.org/10.1177/1545968308331143

4. Rogers JC, Figone JJ. Traumatic quadriplegia: Follow-up study of self-care skills. Arch Phys Med Rehabil. 1980; 61(7):316-21. [PMID:7396683]

5. Knox CC, Engel WH, Siebens AA. Results of a survey on the use of a wrist-driven splint for prehension. Am J Occup Ther. 1971;25(2):109-11. [PMID:5551513]

6. Allen VR. Follow-up study of wrist-driven flexor-hingesplint use. Am J Occup Ther. 1971;25(8):420-22. [PMID:5097917]

7. Shepherd CC, Ruzicka SH. Tenodesis brace use by persons with spinal cord injuries. Am J Occup Ther. 1991;45(1): 81-83. [PMID:2000929]

http://dx.doi.org/10.5014/ajot.45.1.81

8. Sargant C, Braun MA. Occupational therapy management of the acute spinal cord-injured patient. Am J Occup Ther. 1986;40(5):333-37. [PMID:3717269] http://dx.doi.org/10.5014/ajot.40.5.333

9. Moore G. An alternative technique for fabricating flexor hinge hand orthoses using total contact molded plastic finger pieces. Clin Prosthet Orthot. 1986;10(3):115-18.

10. Nickel VL, Perry J, Garrett AL. Development of useful function in the severely paralyzed hand. J Bone Joint Surg Am. 1963;45:933-52. [PMID:14047365]

11. McKenzie MW. The ratchet handsplint. Am J Occup Ther. 1973;27(8):477-79. [PMID:4751192]

12. Anderson MH, Sollars RE. Upper extremities orthotics. Springfield (IL): Thomas; 1965.

13. Barber LM, Nickel VL. Carbon dioxide-powered arm and hand devices. Am J Occup Ther. 1969;23(3):215-25. [PMID:5789486]

14. Engen TJ. Development of externally powered upper extremity orthotic systems. J Bone Joint Surg Br. 1965;47: 465-68. [PMID:14341062]

15. Engen TJ. Restoration of function in upper extremities by external power. Arch Phys Med Rehabil. 1966;47(3):182-89. [PMID:5904922]

16. Lehneis HR. Application of external power in orthotics. Orthot Prosthet. 1968;22(3):34-45.

17. Mulcahey MJ. Upper limb orthoses for the person with spinal cord injury. In: Hsu JD, Michael JW, Fisk JR, editors. AAOS atlas of orthoses and assistive devices. 4th ed. Philadelphia (PA): Mosby/Elsevier; 2008. p. 203-18.
18. Thorsen RA, Occhi E, Boccardi S, Ferrarin M. Functional electrical stimulation reinforced tenodesis effect controlled by myoelectric activity from wrist extensors. J Rehabil Res Dev. 2006;43(2):247-56. [PMID:16847791] http://dx.doi.org/10.1682/JRRD.2005.04.0068

19. Mangold S, Keller T, Curt A, Dietz V. Transcutaneous functional electrical stimulation for grasping in subjects with cervical spinal cord injury. Spinal Cord. 2005;43(1): 1-13. [PMID:15289804] http://dx.doi.org/10.1038/sj.sc.3101644

20. Crema A, McNaught A, Albisser U, Bolliger M, Micera S, Curt A, Morari M. A hybrid tool for reaching and grasping rehabilitation: the ArmeoFES. Conf Proc IEEE Eng Med Biol Soc. 2011;2011:3047-50. [PMID:22254982] http://dx.doi.org/10.1109/IEMBS.2011.6090833

21. Saxena S, Nikolić S, Popović D. An EMG-controlled grasping system for tetraplegics. J Rehabil Res Dev. 1995; 32(1):17-24. [PMID:7760263]

22. Hart RL, Kilgore KL, Peckham PH. A comparison between control methods for implanted FES hand-grasp systems. IEEE Trans Rehabil Eng. 1998;6(2):208-18. [PMID:9631329] http://dx.doi.org/10.1109/86.681187

23. Hamou C, Shah NR, DiPonio L, Curtin CM. Pinch and elbow extension restoration in people with tetraplegia: A systematic review of the literature. J Hand Surg Am. 2009; 34(4):692-99. [PMID:19345872] http://dx.doi.org/10.1016/j.jhsa.2008.12.002

24. Cizmar I, Zalesak B, Pilny J, Drac P, Fialova J. Possible restorations of the upper extremity motion in tetraplegic patients - 5-year clinical experience. Biomed Pap Med Fac Univ Palacky Olomouc Czech Repub. 2006;150(2):313-19. [PMID:17426799]

http://dx.doi.org/10.5507/bp.2006.048

25. Reinholdt C, Fridén J. Rebalancing the tetraplegic wrist using extensor carpi ulnaris-tenodesis. J Hand Surg Eur Vol. 2013;38(1):22-28. [PMID:22777853] http://dx.doi.org/10.1177/1753193412453412

26. Sabine CL, Addison RG, Fischer HK. A plastic tenodesis splint; Preliminary evaluation of a functional brace for a paralyzed hand with effective wrist extensors. J Bone Joint Surg Am. 1965;47:533-36. [PMID:14275174]

27. Bacon G, Olszewski E. Sequential advancing flexion retention attachment: A locking device for the wrist-driven flexor hinge splint. Am J Occup Ther. 1978;32(9):577-79. [PMID:707254]

28. Malick MH, Meyer CM. Manual on management of the quadriplegic upper extremity, using available modular splint and arm support systems. Pittsburgh (PA): Harmarville Rehabilitation Center; 1978. 
29. Sabine C, Sammons F, Michela BJ. Report of development of the RIC plastic tenodysis splint. Arch Phys Med Rehabil. 1959;40:513-15. [PMID:14440560]

30. Rosen WR, McColey JJ, Bowker JH. The team approach to orthotic management in quadriplegia. Clin Prosthet Orthot. 1987;11(4):201-9.

31. Nichols PJ, Peach SL, Haworth RJ, Ennis J. The value of flexor hinge hand splints. Prosthet Orthot Int. 1978;2(2): 86-94. [PMID:152909]

32. Maynard FM Jr, Bracken MB, Creasey G, Ditunno JF Jr, Donovan WH, Ducker TB, Garber SL, Marino RJ, Stover $\mathrm{SL}$, Tator CH, Waters RL, Wilberger JE, Young W. International standards for neurological and functional classification of spinal cord injury. American Spinal Injury Association. Spinal Cord. 1997;35(5):266-74.

[PMID:9160449]

http://dx.doi.org/10.1038/sj.sc.3100432

33. Hislop HJ, Montgomery J, Connolly BH, Daniels L, Worthingham C. Daniels and Worthingham's muscle testing: Techniques of manual examination. 6th ed. Philadelphia (PA): W. B. Saunders; 1995.

34. Mathiowetz V, Kashman N, Volland G, Weber K, Dowe M, Rogers S. Grip and pinch strength: Normative data for adults. Arch Phys Med Rehabil. 1985;66(2):69-74. [PMID:3970660]

35. Pryce JC. The wrist position between neutral and ulnar deviation that facilitates the maximum power grip strength. J Biomech. 1980;13(6):505-11. [PMID:7400178] http://dx.doi.org/10.1016/0021-9290(80)90343-7

36. Perotto AE. Anatomical guide for the electromyographer: The limbs and trunk. 3rd ed. Springfield (IL): Charles C. Thomas; 1994.

37. Stenehjem J, Swenson J, Sprague C. Wrist driven flexor hinge orthosis: Linkage design improvements. Arch Phys Med Rehabil. 1983;64(11):566-68. [PMID:6639320]

38. Johnson H. Radial deviation modification to wrist driven flexor hinge orthosis. J Prosthet Orthot. 1990;2(4):305-8.

39. Bottlang M, Marsh JL, Brown TD. Articulated external fixation of the ankle: Minimizing motion resistance by accurate axis alignment. J Biomech. 1999;32(1):63-70.

\section{[PMID:10050952]}

http://dx.doi.org/10.1016/S0021-9290(98)00143-2

40. Lehneis HR. Brace alignment considerations. Orthot Prosthet Appl J. 1964;18:110-14.

41. Perry J. Prescription principles. In: American Academy of Orthopedic Surgeons, editors. Atlas of orthotics: Biomechanical principles and application. St. Louis (MO): C. V. Mosby Co; 1975. p. 105-29.

42. Brumfield RH, Champoux JA. A biomechanical study of normal functional wrist motion. Clin Orthop Relat Res. 1984;(187):23-25. [PMID:6744724]

43. Irani KD. Upper limb orthosis. In: Braddom RL, Buschbacher RM, Dumitru D, Johnson EW, Matthews D, Sinaki $\mathrm{M}$, editors. Physical medicine and rehabilitation. 1st ed. Philadelphia (PA): Saunders; 1996. p. 321-32.

44. Smith AG. Early complications of key grip hand surgery for tetraplegia. Paraplegia. 1981;19(2):123-26.

[PMID:7220067]

http://dx.doi.org/10.1038/sc.1981.27

Submitted for publication October 18, 2012. Accepted in revised form February 21, 2013.

This article and any supplementary material should be cited as follows:

Kang YS, Park YG, Lee BS, Park HS. Biomechanical evaluation of wrist-driven flexor hinge orthosis in persons with spinal cord injury. J Rehabil Res Dev. 2013; 50(8):1129-38.

http://dx.doi.org/10.1682/JRRD.2012.10.0189

ResearcherID/ORCID: Hyung-Soon Park, PhD: B-33342010

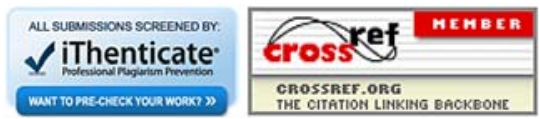


